\title{
Visceral Targets Specify Calcitonin Gene-related Peptide and Substance P Enrichment in Trigeminal Afferent Projections
}

\author{
Kevin Horgan and Derek van der Kooy \\ Neurobiology Research Group, Department of Anatomy, University of Toronto, Toronto, Ontario, Canada M5S 1A8
}

Rat trigeminal ganglion projections to a visceral target (intracranial blood vessels) are enriched in calcitonin generelated peptide (CGRP) and substance $P$ (Sub P) compared to trigeminal ganglion projections to a cutaneous target (the forehead skin). We asked if transplants of a novel visceral target (fetal stomach antrum tissue) into the path of the neonatal rat trigeminal frontal nerve projection to forehead skin would induce neuronal CGRP and Sub P enrichment. By postnatal day (P) 25, the percentage of nerves containing CGRP increased from $14-15 \%$ in the control trigeminal projection to forehead skin to $20-31 \%$ (in different experiments) in the trigeminal projection to transplanted stomach antrum. The percentage of Sub P-containing neurons increased from $10 \%$ in the control forehead skin projection to $22 \%$ in the trigeminal projection to stomach transplants over the same time period. The number of neurons in the trigeminal frontal nerve projection to stomach antrum transplants was not significantly different from the number of frontal neurons projecting to control forehead skin. We suggest that respecification of trigeminal neurons to the CGRP and Sub P phenotype, not selective survival of CGRP- and Sub P-positive afferents, is the mechanism by which stomach antrum induces enrichment of CGRP and Sub P.

A subpopulation of rat trigeminal neurons with cutaneous forehead skin projections also sends a transient axon collateral projection to a visceral target (the cerebral arteries) during early postnatal development. Postnatal maintenance of an axonal projection to a cutaneous target (forehead skin) may be incompatible with a neuron also maintaining a visceral collateral to the cerebral arteries. Neonatal transplants of stomach antrum were made into the path of the trigeminal projection to forehead skin. This tested whether frontal nerve projections to a novel visceral target would protect these individual trigeminal neurons from the normal retraction of their visceral axon collaterals to the cerebral arteries. Fourteen percent of trigeminal neurons in the cutaneous projection to the forehead skin also project to the middle cerebral artery at P5 in control rats. By P90, all of these cells survive and maintain cutaneous forehead skin projections, but almost all have retracted their artery collaterals in control animals. Less than $4 \%$ of trigeminal neurons projecting to transplanted stomach antrum retain their cerebral artery col-

\footnotetext{
Received July 9, 1991; revised Sept. 19, 1991; accepted Oct. 28, 1991.

This work was supported by the Heart and Stroke Foundation of Ontario.

Correspondence should be addressed to Kevin Horgan, Neurobiology Research Group, Department of Anatomy, University of Toronto, Toronto, Ontario, Canada M5S 1A8.

Copyright (C) 1992 Society for Neuroscience $0270-6474 / 92 / 121135-09 \$ 05.00 / 0$
}

lateral projection at P25, the same low percentage of cells retaining artery collaterals in the trigeminal projection to control transplants of forehead skin at this age. Thus, in the trigeminal system, postnatal forehead target phenotype is important in regulating neurotransmitter expression in neuronal afferents, but may not be the critical factor controlling the axon collateral distribution of neurons projecting to multiple peripheral targets.

Interactions between neurons and their peripheral tissue targets influence the development of peripheral nerve projections. In particular, these interactions are important in the regulation of neurotransmitter phenotype in projecting neurons, as well as in the selective stabilization of peripheral neuronal connections that occurs during the developmental cell death and axon retraction period. There is a strong correlation in sensory neurons between neurochemical profile and peripheral target tissue (Marusich et al., 1986a; Philippe and Droz, 1986; Gibbins et al., 1987; McMahon and Gibson, 1987; Molander et al., 1987; O'Brien et al., 1989). In addition, the onset of expression of neurochemical markers has been correlated with initial target innervation (Marusich et al., 1986a; Philippe et al., 1988; Katz and Erb, 1990). In vitro studies have also revealed the induction of neurochemical expression in sensory neurons by coculturc with their targets (Philippe et al., 1988; Weston et al., 1988). Direct tests of target regulation of neurotransmitter phenotype involving de novo growth of the sympathetic sweat gland projection to novel peripheral targets in rats demonstrate that the presence of the sweat gland target is necessary postnatally for the normal expression of cholinergic properties in projecting sympathetic neurons. Moreover, neonatal transplants of sweat gland tissue induce cholinergic properties in a previously noradrenergic sympathetic projection (Shotzinger and Landis, 1988, 1990b). Perhaps the most convincing evidence for target regulation of neurotransmitter phenotype in sensory neurons in vivo has come from studies showing changes in neurotransmitter or neurochemical expression in regenerating sensory neurons after their experimentally manipulated innervation of novel targets (McMahon and Gibson, 1987; McMahon and Moore, 1988; McMahon et al., 1989). Studies analogous to those on target regulation of neurochemical expression in sensory afferent projections demonstrate that changing peripheral target phenotype results in a corresponding change in central projections of afferent neurons. When dorsal root ganglion (DRG) cutaneous afferents are forced to innervate muscle, there is a resultant change in their central projections in the spinal cord; they make novel connections on motoneurons appropriate for their new peripheral target (Smith and Frank, 1987). Further evidence of 
peripheral target regulation of sensory afferent phenotype comes from a study where neonatal exposure of cutaneous afferents to antiserum against NGF (which is present in the target epidermis of these afferents) disrupts the normal cutaneous target specification of afferent receptor subtype (Ritter et al., 1991).

The trigeminal ganglion provides sensory innervation to cutaneous, muscular, and visceral targets in the head. In the rat, trigeminal afferents first reach their peripheral targets between embryonic days (E) 13-15 (Erzurumla and Killackey, 1983). Onset of neurotransmitter expression in trigeminal neurons occurs several days later. Irigeminal cells expressing the neurotransmitter substance $P$ (Sub P) are first seen at E17 in the trigcminal ganglion (Kessler and Black, 1981). Expression of the neurotransmitter calcitonin gene-related peptide (CGRP) in peripheral trigeminal processes is first seen in cerehral blond vessels and olfactory epithelium at E18 (Tsai et al., 1989; Baker, 1990). Trigeminal sensory neurons exhibit distinct neurotransmitter profiles that are characteristic of the peripheral target innervated (Terenghi et al., 1986; Ositelu et al., 1987; O'Connor and van der Kooy, 1988; Edvinsson et al., 1989). In particular, trigeminal visceral projections to the cerebral arteries and cutaneous projections to the forehead skin differ in the percentage of afferent neurons in each projection that contains CGRP and Sub P. Thirty percent of trigeminal cerebral artery afferents contain CGRP, while less than $15 \%$ of trigeminal forehead skin afferents contain CGRP. Similarly, $18 \%$ of the trigeminal cerebral artery-projecting neurons contain Sub $\mathrm{P}$, compared to the less than $10 \%$ of forehead skin trigeminal afferents that contain Sub P (O'Connor and van der Kooy, 1988).

The differences in the CGRP and Sub $P$ profiles of trigeminal projections to the cerebral arteries versus the forehead skin raise the question of the developmental mechanisms regulating neurotransmitter profiles in neurons whose perikarya are intermixed in the opthalmic division of the ganglion but whose peripheral projections differ. The trigeminal visceral projection to the cerebral arteries is characterized by a period of postnatal cell death and axon retraction that results in a loss of more than three-quarters of the neurons in this projection between postnatal day (P) 5 and P90. In contrast, over this same period the trigeminal projection to the forehead skin undergoes no significant cell death or axon retraction from the forehead target (Horgan et al., 1990). There is a correlation between the postnatal regressive events and the adult enrichment in CGRP and Sub $P$ in the trigeminal cerebral artery projection, whereas an absence of regressive events and a comparatively reduced presence of CGRP and Sub P characterize the trigeminal forehead skin projection. This could indicate a causal link between postnatal regressive events in the cerebral artery projection and enrichment of CGRP and Sub P. However, observations in the trigeminal cerebral artery projection reveal that CGRP-containing neurons do not selectively survive at the cerebral artery, nor do CGRP-negative neurons selectively regress from the artery, during the postnatal cell death and axon retraction period in this projection. Rather, maintenance of an artery projection postnatally ensures CGRP enrichment whereas postnatal retraction of artery collaterals results in a respecification of some retracting neurons to the non-CGRP phenotype (Horgan et al., 1990). These results lead to the hypothesis that cerebral arterial targets may directly induce CGRP enrichment in trigeminal afferents. Enrichment of both CGRP and Sub $P$ is seen in visceral afferent projections throughout the body from the trigeminal ganglion and several DRG (Su et al., 1986; Terenghi et al.,
1986; Green and Dockray, 1987, 1988; Molander et al., 1987; O'Connor and van der Kooy, 1988). In particular, the DRG sensory projection to stomach antrum is highly enriched in both CGRP cells and Sub P afferents, with reports of 75-85\% CGRP cells and $50-60 \%$ Sub $P$ cells in this projection (Green and Dockray, 1988). Thus, the first question to be asked in the present study is whether transplants of fetal stomach antrum tissue into the path of neonatal cutaneous trigeminal afferents to the forehead skin will produce an enrichment in CGRP and Sub $\mathbf{P}$ in these neurons.

The effects of target phenotype may extend beyond the regulation of afferent neurotransmitter profile to the regulation of afferent conncetivity. Axon retraction in the trigeminal system appears to play a significant role in refining peripheral connectivity during postnatal development. In neonatal animals, approximately $15 \%$ of the neurons afferent to the forehead skin (a cutaneous target) also project to the cerebral arteries (a visceral target). By P90, almost all of these cells have retracted their artery collaterals but have retained their forehead projection and do not die. This indicates that axon retraction facilitates the functional segregation of visceral and cutaneous projections in the trigeminal system (O'Connor and van der Kooy, 1986; Horgan et al., 1990). It has been suggested that different functional classes of sensory neurons (i.e., cutaneous, visceral, and proprioceptive) may have distinct neurotrophic requirements (Davies, 1987). We hypothesized that visceral and cutaneous trigeminal targets release different trophic factors that selectively support only the appropriate class of sensory afferent during development. In this way, single trigeminal neurons would be constrained to project to only visceral or only cutaneous targets by means of regressive events during development. Support for this idea comes from a study where neurotrophic factors from the corneal epithelium selectively enhance survival of only a subpopulation of trigeminal opthalmic division neurons (corneal epithelium is a normal target for opthalmic afferents) (Chan and Haske, 1981). Another study also shows that neural crestderived cutaneous trigeminal afferents are responsive to NGF (which is present during development in trigeminal cutaneous targets) but show no responsiveness to separate neurotrophic factors derived from other, noncutaneous targets (Davies and Lindsay, 1984). Thus, the second question to be asked in the present study is whether transplants of fetal stomach antrum tissue into the path of cutaneous trigeminal afferents to the forehead skin will prevent the normal axon retraction of cerebral artery collatcrals by these same ncurons.

\section{Materials and Methods}

The first transplant paradigm. The offspring of pregnant albino Wistar rats were used in this experiment. Rat pups were recorded as P0 on day of birth. Stomach antrum tissue from embryonic day (E) 18 rat fetuses was dissected out under sterile conditions and kept in Dulbecco's modified Eagle's medium (D-MEM) containing 20\% calf serum until time of transplantation. Stomach antrum tissue was chosen as donor tissue because a large percentage of DRG afferents projecting to this target contain CGRP and Sub P (Green and Dockray, 1988). E18 was chosen as an optimum age for donor tissue, because this is $3-4 \mathrm{~d}$ after DRG afferents first contact their peripheral targets and $1 \mathrm{~d}$ after DRG afferents first show evidence of neurotransmitter expression (Kessler and Black, 1981; Altman and Bayer, 1982). P0 rat pups were anesthetized using cold temperatures. The right forehead skin flap was opened and the frontal nerve dissected out and cut just distal to the supraorbital foramen. Fetal stomach antrum tissue was wrapped around the proximal stump of the frontal nerve, and the transplanted tissue was surrounded by Gelfoam to prevent it from moving. Animals were sutured and then rewarmed until they recovered. Control animals received frontal nerve 
cuts and applications of Gelfoam. In both of these procedures, sufficient true blue (TB) was applied to ensure labeling of the entire trigeminal projection to the transplant tissue. At $\mathrm{P} 22$, animals were anesthetized using sodium pentobarbital $(65 \mathrm{mg} / \mathrm{kg})$, and the retrograde fluorescent axonal tracer TB was applied to transplanted stomach antrum in the experimental group and to the forehead skin in controls. In controls, powdered TB was directly applied to the frontal nerve after it was sectioned at P22 and then covered by Gelfoam. In transplant animals, either powdered TB was applied directly to the stomach tissue and then covered by Gelfoam, or (in larger transplants) injections of less than 1 $\mu \mathrm{l}$ of $5 \%$ TB were made directly into the lumen of the transplant tissue. At P22, transplants were generally fluid-filled spheres of tissue $1 / 2$ to 2 $\mathrm{cm}$ in diameter that were clearly identifiable by distinctive surface appearance, plentiful vascularization, and dense innervation. At P25, animals were killed. This age was chosen because previous studies in the trigeminal system indicate that a variety of developmental events are complete by this age (O'Connor and van der Kooy, 1989; Tsai et al., 1989).

The second transplant paradigm. The first transplant paradigm was modified in several ways to test whether technical constraints limited the stomach antrum-induced CGRP enrichment in trigeminal afferents in the first transplant paradigm. To control for host animal immune reaction against transplanted tissue, the offspring of inbred Fischer 344 rats were used both as donors and recipients. Control animals received E18 forehead skin tissue transplants wrapped around the proximal stump of the cut right frontal nerve (instead of just frontal nerve cuts), to control for the nonspecific effects of transplanting any type of tissue. Cutting the frontal nerve may precipitate cell death in frontal nerve afferents, and selective cell death could conceivably produce changes in neurotransmitter profiles. Therefore, the effect of nerve cut was controlled for in both control and experimental groups by cutting and not cutting the nerve in half of each group. To limit tracer spread, only small applications of fast blue (FB) were made to the transplanted tissues. These applications were immediately covered by a small square of Parafilm, and then Vaseline was smeared over and around the application site to prevent leakage. At death, transplant tissue was removed from animals and was fixed and sectioned (as described below) to verify tracer spread. TB was the tracer used in the first transplant paradigm, and FB was used in the second paradigm. The results with these tracers were treated as interchangeable, since our previous work has shown no difference in retrograde labeling in the rat trigeminal system with TB and FB (Horgan et al., 1990).

The adult DRG sensory projection to stomach antrum. Male adult Fischer 344 inbred rats were anesthetized using sodium pentobarbital $(65 \mathrm{mg} / \mathrm{kg})$. The abdominal cavity was opened and the ventral aspect of the stomach was exposed. A small powdered FB application to stomach antrum was covered in Gelfoam, and then stomach was folded over the application site and carefully stitched to seal the application site. At least $4 \mathrm{~d}$ post-FB application preceded death to allow for retrograde transport of FB.

Postnatal retraction of cerebral artery collaterals in trigeminal frontal nerve-projecting cells. Offspring of Fischer 344 inbred pregnant rats were used in this experiment. PO transplants in both control and experimental groups were made exactly as described in the modified transplant paradigm. At P22, the animals were anesthetized using sodium pentobarbital $(65 \mathrm{mg} / \mathrm{kg})$. From a parietotemporal approach, the right middle cerebral artery (MCA) was isolated just ventral to the rhinal sulcus using a Zeiss operating microscope. A small amount of powdered TB or FB was applied directly to the artery and then covered by one or two pieces of Gelfoam. Animals were sutured and allowed to recover. At P24, the animals were again anesthetized and the retrograde fluorescent tracer diamidino yellow (DY) was applied to the transplanted tissues and covered by a small square of Parafilm and by Vaseline. At P25, animals were killed. P25 was chosen because postnatal regression in the trigeminal MCA projection is largely complete by this age (O'Connor and van der Kooy, 1989).

Tissue processing and immunocytochemistry. At the time of death, animals were deeply anesthetized with sodium pentobarbital and decapitated. The right trigeminal ganglion and DRG from thoracic levels 9-12 (when assaying the normal CGRP presence in DRG afferents to stomach antrum) were removed from the rats, washed in D-MEM, and then cultured in D-MEM containing $10^{-4} \mathrm{M}$ colchicine for $2-3 \mathrm{hr}$ (neonatal ganglia) or 5-6 hr (P25 and adult ganglia). Colchicine pretreatment in vitro improves visualization of neurotransmitter immunohistofluorescence without increasing the overall number of transmitter-positive cells identified in sensory ganglia (O'Connor and van der Kooy, 1988), and was used here to reduce variability in the scoring of CGKP- and Sub P-positive cells. Ganglia were then immersed in $4 \%$ paraformaldehyde in $0.1 \mathrm{M}$ phosphate-buffered saline (PBS) for $24 \mathrm{hr}$ at $4^{\circ} \mathrm{C}$ and then transferred to $20 \%$ sucrose for another $24 \mathrm{hr}$ at $4^{\circ} \mathrm{C}$. Twenty-four micrometer sections were cut on the cryostat. For CGRP immunostaining, ganglion sections were incuhated at $4^{\circ} \mathrm{C}$. for $24 \mathrm{hr}$ with primary antiserum to CGRP (Penninsula, against Tyr-CGRP) diluted to 1:300 in $0.1 \mathrm{~m}$ PBS, containing $1 \%$ normal goat serum (NGS) or normal horse serum (NHS) and $0.3 \%$ Triton X-100. After primary incubation, the ganglia were rinsed twice (rinsing solution: 0.1 m PBS containing $1 \%$ NGS or NHS and $0.3 \%$ Triton X-100) and then incubated at room temperature in goat or sheep anti-rabbit IgG conjugated with fluorescein isothiocyanate (Dimension) diluted 1:50. After a final rinse with $0.1 \mathrm{M}$ PBS, sections were mounted on gel-coated slides. For Sub P immunostaining, ganglion sections were incubated at $4^{\circ} \mathrm{C}$ for $24 \mathrm{hr}$ with a monoclonal antiserum to Sub P (Sera Lab) diluted to 1:50 in 0.1 M PBS containing $1 \%$ NGS or NHS and 3\% Triton X-100. After primary incubation, sections were rinsed two to three times in the rinsing solution and then incubated for $60 \mathrm{~min}$ at room temperature in a biotinylated horse anti-mouse IgGr (Dimension) diluted 1:50. After rinsing, sections were then transferred to a 1:50 avidin-conjugated rhodamine isothiocyanate (Dimension) solution for another $60 \mathrm{~min}$ at room temperature. After a final rinse in 0.1 м PBS, sections were mounted on gel-coated slides. Sections were counted under an epifluorescent Leitz microscope using a $360 \mathrm{~nm}$ filter for TB, FB, and DY labeling, a $470 \mathrm{~nm}$ filter for CGRP fluorescein staining, and a $550 \mathrm{~nm}$ filter for Sub $\mathbf{P}$ rhodamine staining. Retrogradely labeled cells were counted in all sections or every second section for CGRP, and in every second section for Sub $P$, through the ganglia. Neurotransmitter-positive cells were counted among the TB and FB retrogradely labeled populations. All counts were performed by the same experimenter, who was blind as to whether the tissue was from experimental or control ganglia. All counts were corrected using the method of Abercrombie (1946). Absorption controls demonstrated the specificity of the CGRP and Sub P antisera by the abrogation of immunostaining in ganglion sections incubated in primary CGRP antiserum or Sub $P$ antiserum absorbed with the CGRP peptide (Penninsula) or with the Sub P peptide (Sigma), respectively.

\section{Results}

CGRP enrichment by stomach antrum transplants in the first transplant paradigm

Fetal stomach antrum transplants produced a significant $(t=$ $2.9, p<0.05 ; n=19$ control, $n=18$ experimental) enrichment in CGRP-positive afferents, increasing the percentage of CGRPcontaining cells from $15 \%$ in control frontal nerve projections to the forehead skin to $20 \%$ in experimental frontal nerve projections to transplanted stomach antrum (Fig. $1 A$ ). This $5 \%$ enrichment in the percentage of cells containing CGRP (approximately $10-15$ of the neurons giving rise to this projection) occurred in the absence of any significant $(t=0.16, p>0.05$; $n=19$ control, $n=18$ experimental) change in the number of labeled frontal nerve-projecting cells in transplanted animals compared to controls (Fig. $1 B$ ). This result suggests that the CGRP enrichment in transplanted animals was due to the respecification of non-CGRP-positive trigeminal neurons to the CGRP phenotype by the stomach antrum target, rather than to the selective survival of CGRP-containing trigeminal stomach antrum afferents. An example of CGRP-immunoreactive staining is shown in Figure 2.

\section{Sub P enrichment by stomach antrum transplants in the first transplant paradigm}

Fetal stomach antrum transplants also produced significant ( $t$ $=2.2, p<0.05 ; n=8$ control, $n=8$ experimental) enrichment in Sub P-positive trigeminal afferent projections, increasing the pcrcentage of Sub $\mathrm{P}$-containing cells from $10 \%$ in control frontal nerve projections to forehead skin to $22 \%$ in experimental fron- 

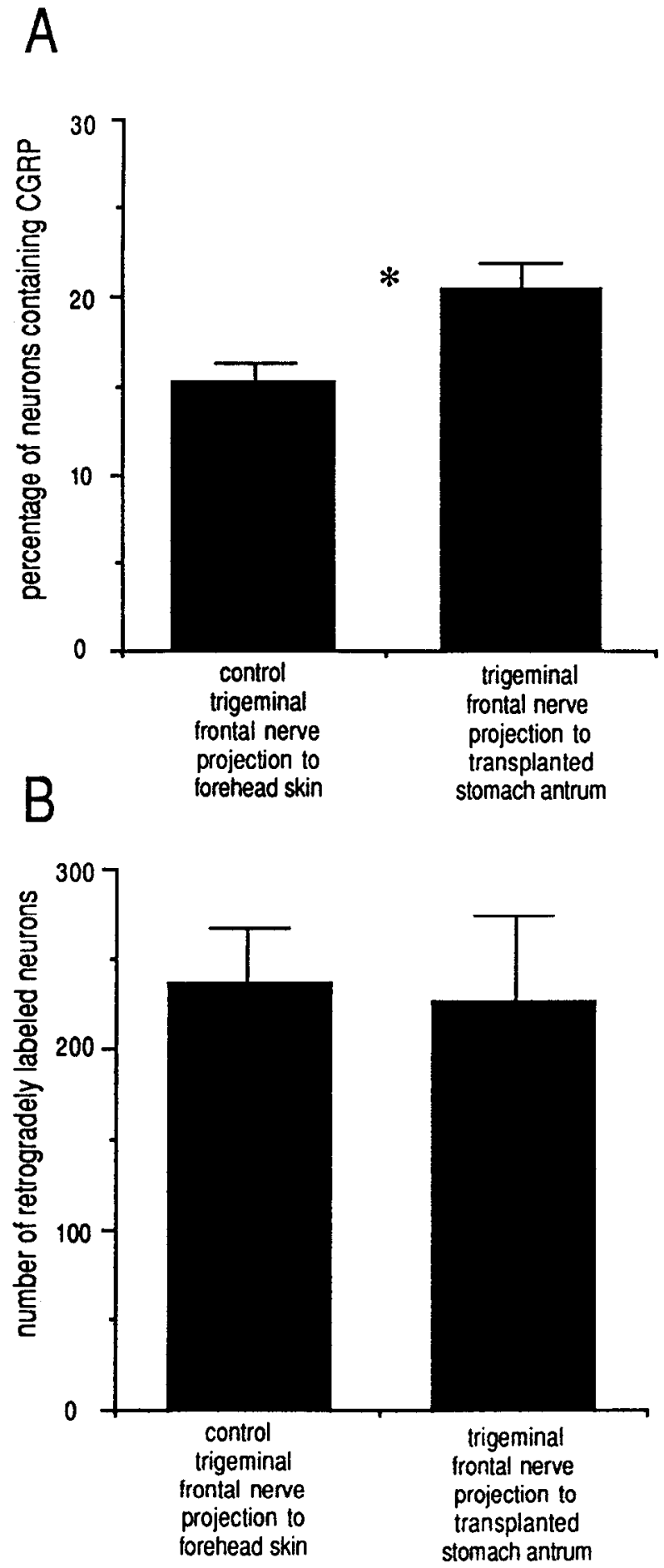

Figure 1. A, Percentage of retrogradely labeled trigeminal neurons that were CGRP positive in the frontal nerve projection to neonatal transplants of fetal stomach antrum tissue and to normal control forehead skin. $B$, Number of retrogradely labeled trigeminal neurons at P25 in the same projections described in $A$. Data represent means \pm SEM. * indicates $p<0.05$ for that comparison.

tal nerve projections to stomach antrum transplants (Fig. $3 A$ ). This enrichment involved approximately 10-15 of the ncurons giving rise to this projection. The total number of labeled projecting cells was not significantly $(t=0.8, p>0.05 ; n=8$ control, $n=8$ experimental) different in transplanted animals compared to control animals (Fig. $3 B$ ). This again suggests that Sub $\mathrm{P}$ enrichment by stomach antrum targets involves respecification of projecting non-Sub $P$ neurons to the Sub $P$ phenotype, rather than selective survival of Sub $\mathrm{P}$-containing afferents.

\section{CGRP enrichment by stomach antrum transplants in the second transplant paradigm}

If targets completely control neurotransmitter phenotype in afferent primary sensory neurons, then the report of $75-85 \%$ CGRP-positive cells in the DRG afferent projection to stomach antrum (Green and Dockray, 1988) suggests that the stomach antrum-induced CGRP enrichment in trigeminal afferents is incomplete, perhaps limited by technical constraints in the first paradigm. To investigate this further, several modifications to the transplant paradigm were introduced. Smaller applications of retrograde tracer were used (immediately covered by a combination of Parafilm and Vaseline) to prevent tracer spread to skin surrounding the transplanted tissue, since retrograde tracer uptake by trigeminal nerve fibers in the surrounding skin (low in CGRP) may have masked a greater increase in CGRP enrichment in neurons specifically projecting to transplanted stomach antrum. Note that this application procedure makes impossible any direct comparison of absolute neuronal survival in trigeminal projections to experimental and control transplant tissues since size and position of tracer application were not uniform in this paradigm (as verified by postmortem examination of transplanted tissue). Inbred Fischer 344 rats were employed as host animals and for tissue donors to control for the possibility that an immune reaction in the host animal against transplanted tissue might attenuate the induction of CGRP enrichment in trigeminal afferents. Finally, the percentage of CGRPcontaining cells in the normal DRG projection to stomach antrum was assayed (using the same antibodies to CGRP and the same retrograde tracer labeling protocol as in the transplant paradigm) to provide a control comparison for the stomach antrum-induced CGRP enrichment in trigeminal afferents. Two other controls were introduced to the transplant paradigm. First, to control for the nonspecific effects of transplanting any type of tissue, E18 forehead skin transplants (instead of no tissue) were undertaken in the control animals. Second, half of the animals in both the experimental and control groups were given transplants of stomach antrum and forehead skin, respectively, without first cutting the frontal nerve. This attempted to control (1) for axotomy-induced cell death, which might in itself change the neurotransmitter profile of a projection if there is any specificity in which neurons die, and (2) more importantly, for axotomy-induced changes in neurotransmitter expression that are independent of cell death (Savy et al., 1981; Chiaia et al., 1987; White et al., 1990).

In the second transplant paradigm, the trigeminal projection to transplanted stomach antrum was $31 \%$ CGRP positive at P25. This was a significant $(t=2.7, p<0.05 ; n=7$ experimental, $n=10$ control) increase in the percentage of cells containing CGRP in comparison to the 14\% CGRP-positive cells in the trigeminal projection to control forehead skin transplants at this age (Fig. 4). It was not possible to assess whether neuronal survival was affected by the transplant procedure in the second transplant paradigm (see above); however, results from the first transplant paradigm indicate that there is no significant change in the number of frontal nerve-projecting neurons in response to transplant tissue (Fig. $1 B$ ). Thus, we suggest that the induction of CGRP enrichment by stomach antrum transplants in the 


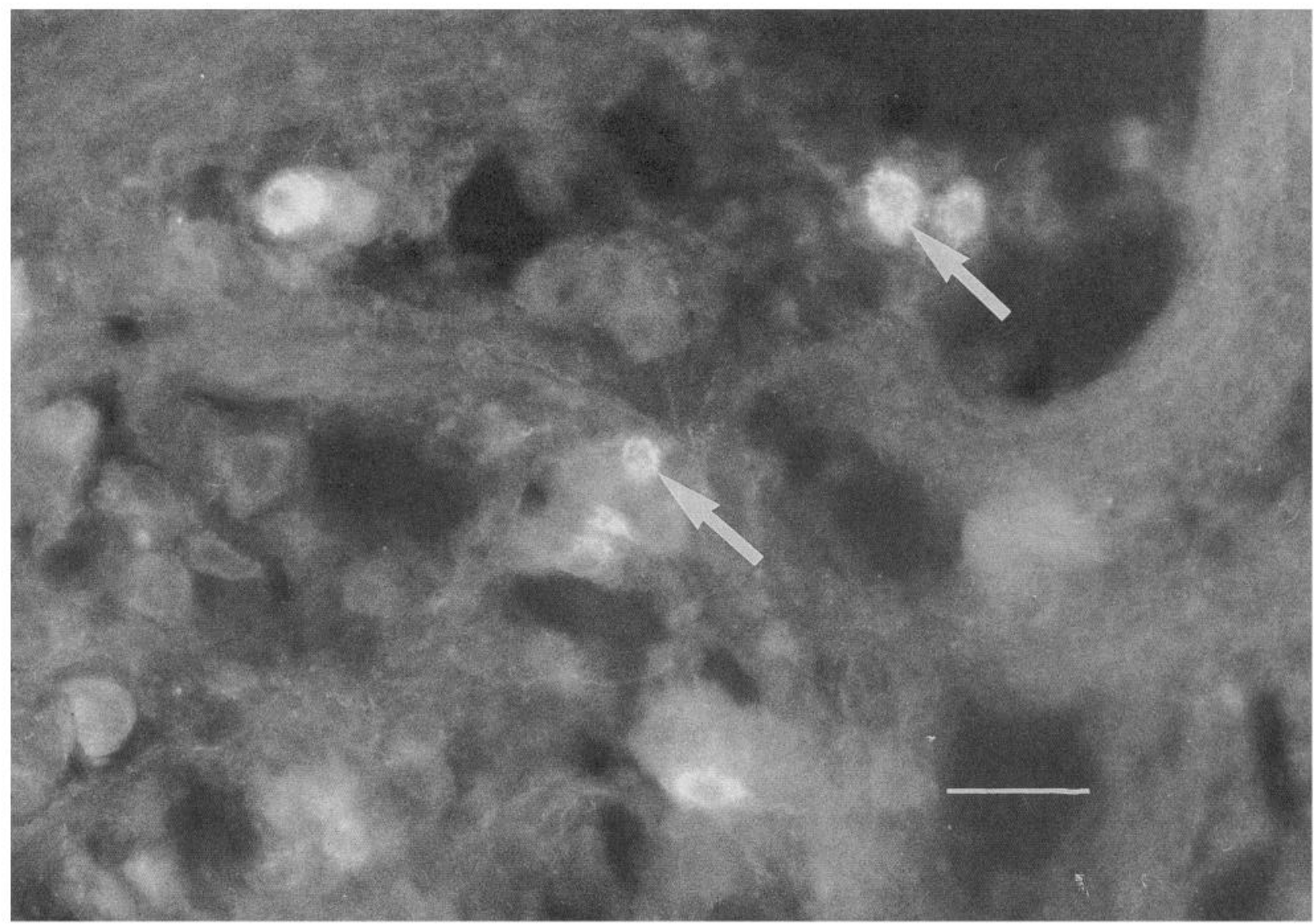

Figure 2. Photomicrograph of a colchicine-treated section of rat trigeminal ganglion removed at the approximate time that neonatal age transplants were made, and stained with an antiserum to CGRP and fluorescein isothiocyanate-conjugated secondary antibodies. Cell bodies were scored positive on the basis of staining intensity, characteristic color, and granular appearance of the staining in the cytoplasm of labeled cells. Arrows point to two of the several CGRP-immunoreactive cell bodies in the field. Scale bar, $50 \mu \mathrm{m}$.

present paradigm is due to respecification of projecting nonCGRP neurons to the CGRP phenotype rather than selective survival of CGRP-positive trigeminal neurons afferent to transplanted stomach antrum. The $14 \%$ CGRP presence in control projections to forehead skin indicates that there is no nonspecific tissue transplant effect involved in the stomach antrum-induced CGRP enrichment. In the control animals, not all of the skin transplants survived until death at P25. However, there was no significant ( $t=0.77, p>0.05 ; n=4$ skin transplant survival, $n=6$ no skin transplant survival) difference in the percentage of CGRP-containing cells in the control group depending on whether the skin transplant survived, nor were there significant effects on CGRP percentages due to cutting frontal nerve branches in the experimental group $(t=1.27, p>0.05 ; n=4$ cut, $n=$ 3 no cut) or in the control group ( $t=1.5, p>0.05 ; n=4$ cut, $n=6$ no cut). This indicates that neither selective, axotomyinduced cell death nor axotomy-induced changes in neurotransmitter expression are likely to be involved in stomach antrum target-induced enrichment of CGRP in trigeminal afferents. In the present experiments, nerve cuts were made distally, just prior to terminal arborizations of the frontal nerve into the forehead skin. Perhaps this distal axotomy is the reason no cell death or neurotransmitter-specific disruptions were seen in response to axotomy, since axotomy-induced disruptions are attenuated the more distal the cut is made (Lieberman, 1974).
In the normal adult DRG projection to stomach antrum, $30 \%$ of the retrogradely labeled cells contained CGRP, a level of enrichment similar to that seen in the trigeminal projection to transplanted stomach antrum tissue (Fig. 4). This suggests that in our paradigm a visceral target (stomach antrum) is able to induce CGRP enrichment (by P25) in the previously cutaneous trigeminal forehead skin projection to the same degree as is seen in the normal visceral DRG projection to stomach antrum at maturity.

\section{The retraction of collateral afferent projections in response to stomach antrum transplants}

At P5, 14\% of the neurons comprising the cutaneous frontal nerve projection to the forehead skin have axon collaterals to the MCA. By P90, less than $1 \%$ of trigeminal forehead skinprojecting neurons retain MCA collaterals, but all of these trigeminal neurons survive and retain their forehead skin collateral projections at maturity (O'Connor and van der Kooy, 1986; Horgan et al., 1990). In animals with stomach antrum transplants placed into the path of previously cutaneous frontal nerve afferents, visceral collateral retraction from the cerebral artery in these frontal nerve afferents appeared to be unaffected. At P25, less than $4 \%$ of the frontal neurons projecting to transplanted stomach antrum retained MCA collaterals. This value was not significantly $(t=0.16, p>0.05 ; n=6$ control, $n=4$ 

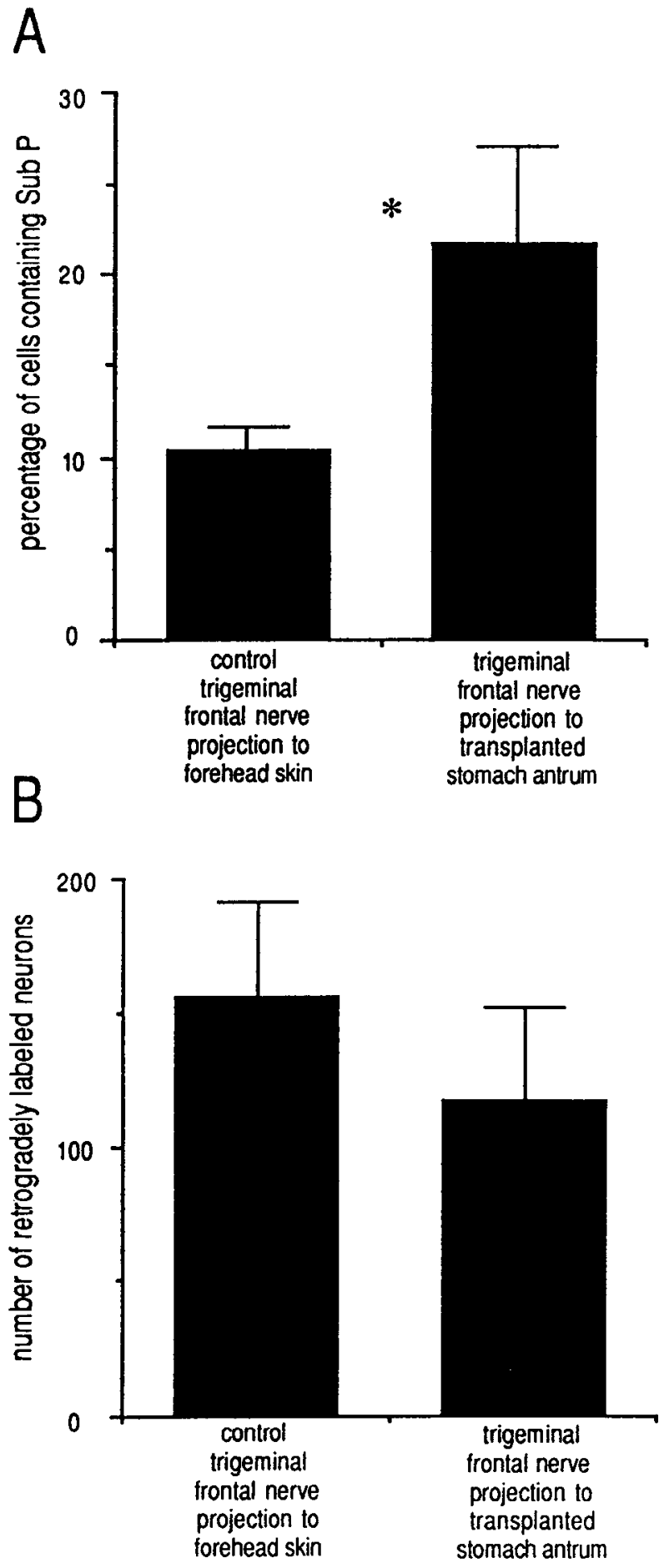

Figure 3. A, Percentage of retrogradely labeled trigeminal neurons that were Sub $\mathbf{P}$ positive in the frontal nerve projection to neonatal transplants of fetal stomach antrum tissue and to normal control forehead skin. $B$, Number of retrogradely labeled trigeminal neurons at P25 in the same projections described in $A$. Data represent means \pm SEM. * indicates $p<0.05$ for that comparison.

experimental) different from the less than $4 \%$ of the control frontal ncurons projecting to transplanted forehead skin that retained MCA collaterals at P25 (Fig. 5). Thus, the visceral cerebral artery collaterals of these trigeminal neurons retract regardless of whether the members of this subpopulation of the frontal nerve projection maintain a cutaneous projection to the

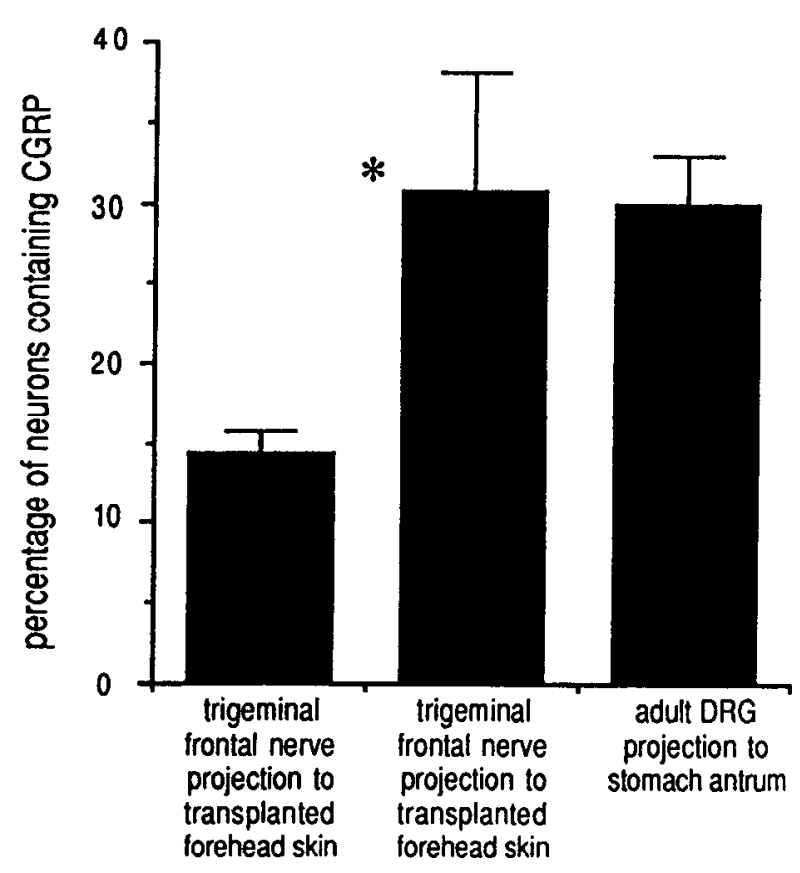

Figure 4. Percentage of retrogradely labeled trigeminal neurons that were CGRP positive in the frontal nerve projection to neonatal transplants of fetal stomach antrum (middle) and to neonatal transplants of fetal forehead skin (left $t)$ in the second transplant paradigm. The bar on the right shows the percentage of retrogradely labeled DRG neurons that were CGRP positive in the normal adult projection to stomach antrum. Data represent means \pm SEM. ${ }^{*}$ indicates $p<0.05$ for that comparison.

forehead skin or maintain a visceral projection to stomach antrum transplanted to the forehead. This indicates that target phenotype does not effect the segregation of the visceral and cutaneous projections of single trigeminal neurons. In addition, these results indicate that the majority of the postnatal collateral retraction from the MCA occurs in forehead-projecting trigeminal neurons by $\mathrm{P} 25$.

\section{Discussion}

These transplant studies suggest that peripheral targets can regulate the neurotransmitter phenotype of projecting sensory neurons. A neonatal visceral transplant of fetal stomach antrum induced CGRP and Sub P enrichment in trigeminal ganglion neurons afferent to this visceral target. Percentage CGRP expression increased from $14 \%$ of the control trigeminal neurons projecting to forehead skin up to as much as $31 \%$ of the trigeminal neurons projecting to transplanted stomach antrum (when immune reactions and tracer application size were controlled). Similarly, Sub P increased from $10 \%$ of the control projection to forehead skin to $22 \%$ of the trigeminal frontal nerve projection to transplanted stomach antrum. Approximately $30 \%$ CGRP-positive neurons are present both in the mature DRG projection to stomach antrum and in the P25 trigeminal projection to transplanted stomach antrum. This suggests that the trigeminal and DRG neuronal populations may offer substrates of similar lability with respect to CGRP expression.

The presence of approximately $30 \%$ CGRP-positive neurons observed in both the trigeminal projection to transplanted stomach antrum and the DRG projection to stomach antrum is markedly lower than the 75-85\% CGRP-positive afferents in the normal DRG sensory innervation to stomach antrum reported 
in another study (Green and Dockray, 1988). Similarly, the $22 \%$ Sub $\mathrm{P}$-containing neurons measured in the trigeminal projection to transplanted stomach antrum is a much lower figure than the $50-60 \%$ Sub $P$ reported in the normal adult DRG innervation of stomach antrum in the previous study (Green and Dockray, 1988). In the second transplant paradigm, we assayed CGRP immunoreactivity in the DRG projections to the stomach antrum using a variety of antisera to CGRP [including the antiserum used in the previous report of very high percentages of CGRP-positive DRG afferents (Green and Dockray, 1988)]. We found that our standard antiserum (Penninsula) showed superior results both in terms of the percentages of retrogradely labeled cells containing CGRP and in terms of CGRP staining intensity per cell. We suggest that different experimental protocols and different scoring criteria may be responsible for the discrepancies in both CGRP and Sub P presence in stomach antrum afferents.

The range of CGRP expression varies from $10 \%$ of the neurons in the normal trigeminal nerve projection to the forehead skin to approximately $30 \%$ of the ncurons in threc projections (the normal trigeminal projection to the cerebral arteries, the normal DRG projection to stomach antrum, and the experimental trigeminal projection to stomach antrum) (O'Connor and van der Kooy, 1989; Horgan et al., 1990; present results). This bounded range raises the possibility that there are intrinsic limits to CGRP expression in sensory afferent populations. Perhaps only a subpopulation of sensory neurons is capable of expressing CGRP, thus establishing an upper limit to CGRP expression in sensory projections. Furthermore, some of the neurons within this subpopulation may be committed to express CGRP early in development, thus establishing a lower limit to CGRP expression in sensory projections postnatally. Evidence for limits on the plasticity of CGRP expression in trigeminal neurons comes again from work documenting CGRP expression in the subpopulation of trigeminal neurons with neonatal projections both to the cerebral arteries and to the forehead skin. In the neonate, $20 \%$ of these neurons express CGRP, whereas in the adult (after almost all of these neurons have retracted their axon collaterals from the cerebral artery) only $16 \%$ express CGRP. The $16 \%$ CGRP expression at P90 in this subpopulation of neurons now solely projecting to the forehead skin is still significantly higher than the 10\% CGRP-positive neurons in the overall population of trigeminal neurons projecting solely to the forehead skin at this time (Horgan et al., 1990). This suggests an incomplete loss of CGRP expression in this subpopulation of trigeminal forehead-projecting neurons with neonatal cerebral artery collateral projections. This may be due to the early developmental commitment of some of this subpopulation of trigeminal neurons to CGRP expression. Further evidence for the idea that sensory neuron subpopulations may be committed early to the expression of a particular neurochemical phenotype comes from in vitro studies using the monoclonal antibody SN 1 , which identifies an epitope on the cell surface of cutaneous sensory afferents. In these studies, SNI expression has been identified on a subpopulation of newly postmitotic sensory afferents put into culture prior to peripheral target contact (Marusich et al., 1986b; Marusich and Weston, 1988). Evidence that there may be upper limits to the proportion of sensory neurons expressing a certain neurochemical phenotype comes from studies on calbindin. This calcium-binding protein has been shown to be expressed in only A1 and B1 DRG cell types (Philippe and Droz, 1988), and it appears that only $15 \%-20 \%$ of DRG afferents are calbindin positive (Philippe and Droz, 1988). Fur-

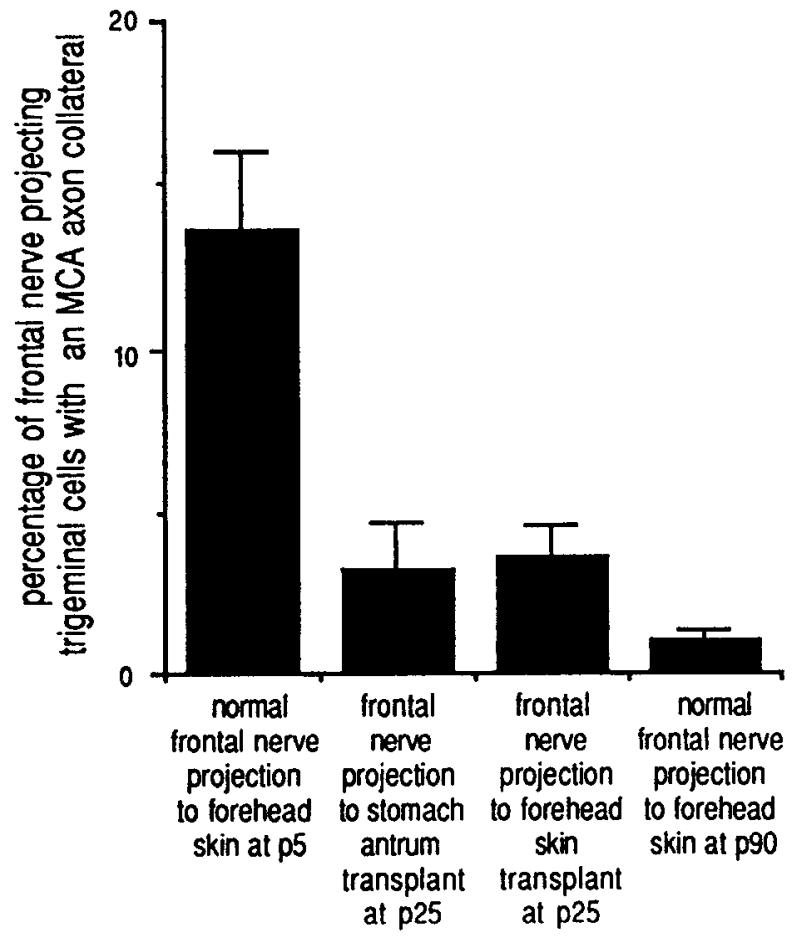

Figure 5. Percentage of frontal nerve-projecting trigeminal cells that possess an axon collateral to the MCA in the normal frontal nerve projection at P5 (left bar; data from Horgan et al., 1990), in the frontal nerve projection to neonatally transplanted fetal stomach antrum or fetal forehead skin (middle bars), and in the normal frontal nerve projection at P90 (right bar; data from O'Connor and van der Kooy, 1986). Data represent means \pm SEM

thermore, induction of calbindin expression in culture by muscle extract (muscle is a target enriched in calbindin-positive DRG affcrents) does not excced $10 \%$ of the cultured ncurons even at saturating concentrations (Barakat and Droz, 1989).

CGRP is enriched in sensory nerve projections to visceral targets throughout the body (Su et al., 1986; Terenghi et al., 1986; Green and Dockray, 1987, 1988; Molander et al., 1987; O'Connor and van der Kooy, 1988). The vasculature (a visceral target) providing the blood supply in the forehead skin could be a specific target for the 10\% CGRP-positive cells in the trigeminal cutaneous projection to the forehead skin, since some CGRP-positive sensory fibers have been reported to course along blood vessels in the dermis of hairy skin in the rat (Kruger et al., 1989). However, the distribution of CGRP-positive sensory nerve fibers is not limited to the vasculature of hairy skin, since CGRP-positive terminations have also been reported in association with hair follicles and as free endings in the epidermis (Kruger et al., 1989; Shotzinger and Landis, 1990a). Although projections to the skin vasculature may account for some of the CGRP presence in this trigeminal cutaneous projection, CGRPpositive trigeminal fibers also appear to terminate on nonvisceral elements in the forehead skin.

The molecular nature of the signals present in stomach antrum tissue that specify CGRP and Sub P expression is unknown at present. In vitro studies on sympathetic neurons reveal several molecules that have been at least partially characterized including cholinergic differentiation factor, membrane-associated neurotransmitter-stimulating factor, ciliary neurotrophic factor, and a low molecular weight protein(s) isolated from sweat gland tissue in rats. All of these molecules induce expression of $\mathrm{ACh}$, 
reduce expression of catecholamines in sympathetic neurons, and have, to a varying degree, effects on Sub P, somatostatin, vasoactive intestinal peptide, and neuropeptide $Y$ expression in sympathetic neurons (Nawa and Patterson, 1990; Rao and Landis, 1990; Rao et al., 1990). In the first transplant paradigm, the similar level of enrichment of CGRP and Sub P in stomach antrum-projecting trigeminal neurons in experimental animals may indicate a common stomach antrum signal for the induction of CGRP and Sub $P$ in projecting trigeminal neurons. CGRP and Sub $P$ have previously been shown to colocalize together in some single sensory neurons (Gibbins et al., 1985; Lee et al., 1985; Su et al., 1986). Evidence consistent with the idea of a single, target-derived signal regulating expression of two distinct neurochemicals comes from an in vitro study revealing a proportionate rise in Sub $\mathrm{P}$ and $\mathrm{ACh}$ in sympathetic neurons after exposure to a variety of conditioned media from different peripheral tissues (Nawa and Sah, 1990). However, previous work on the trigeminal projection to the cerebral artery indicates that this projection is characterized by a CGRP-positive, Sub $\mathrm{P}$-negative subpopulation of afferents ( $\mathrm{O}^{\circ} \mathrm{Connor}$ and van der Kooy, 1988). Thus, it is possible that at least some peripheral targets can induce CGRP expression independent of Sub P in trigeminal neurons.

Modification of neurotransmitter phenotype appears to involve a developmental mechanism independent of the mechanisms underlying axon collateral rearrangement in the same trigeminal sensory neurons. Neonatal transplants of a visceral target (stomach antrum) into the path of cutaneous afferents to the forehead skin do not prevent normal postnatal retraction of the axon collaterals of a subpopulation of these same neurons from another visceral target, the cerebral arteries. Developmental influences other than the contrasting visceral versus cutaneous target phenotypes of this neuronal subpopulation, projecting neonatally to both cerebral artery and forehead skin, must determine the normal retraction of their axon collaterals from the cerebral artery.

Previous studies in this system provide evidence that local competition may be the most important variable regulating axon retraction at the cerebral artery ( $\mathrm{O}^{\prime}$ Connor and van der Kooy, 1989). Strictly local target regulation of afferent survival would suggest that in single neurons with axon collaterals both to the cerebral arteries and to the forehead skin, survival of these two collateral projections would be regulated independently of one another. During normal postnatal development, MCA-projecting afferents from neurons with forehead skin collateral projections are at a competitive disadvantage for survival at the MCA in comparison to neurons projecting only to the cerebral arteries. At P5, MCA-projecting trigeminal neurons with forehead skin collateral projections make up $25 \%$ of the overall MCA projection (Horgan et al., 1990), whereas by maturity trigeminal neurons with forehead skin collateral projections make up less than $3 \%$ of the overall MCA projection (O'Connor and van der Kooy, 1986,1989 ). Local interactions at the MCA may not be the only factor regulating postnatal maintenance of axon collaterals at the MCA.

In neurons projecting to the MCA and to the forehead skin neonatally, postnatal maintenance of a forehead skin projection may itself be important in the selective retraction of axon collaterals to the MCA. A major developmental difference between the trigeminal projection to the cerebral arteries and the trigeminal projection to the forchead skin is the absence of detectable postnatal cell death or axon retraction from the forehead target in the forehead skin projection, contrasting with the large amount of axon retraction and cell death that characterizes the trigeminal cerebral artery projection (O'Connor and van der Kooy, 1986; Horgan et al., 1990). An excess of local neurotrophic factor or target space at the cutaneous forehead target and a limiting amount of trophic factor or target space at the visceral cerebral artery target may underlie the normal development of trigeminal neurons with dual projections neonatally to both the cerebral arteries and the forehead skin. It has been suggested that any given neuron has a limited ability to arborize, and thus an important factor involved in the remodeling of a single neuron's initial pattern of connectivity is competition between sibling neurites of the same nerve cell for limited intracellular constituents necessary to sustain a limited amount of axonal arborization (Smallheiser and Crain, 1984; Saffran and Crutcher, 1990). Single trigeminal neurons with projections to both the forehead skin and cerebral arteries neonatally may be at a competitive disadvantage when competing for survival at the cerebral artery postnatally because of the success of their axon collateral branches in surviving at the forehead skin. From this point of view, transplants of stomach antrum in the place of the forehead skin would not change the competitive disadvantage of cerebral artery collaterals of single trigeminal neurons relative to their sibling frontal nerve collaterals, because transplanted stomach antrum would still provide an excess of the local survival factor or target space for the frontal nerve collaterals.

The visceral phenotype of a transplanted target dictates CGRP and Sub $\mathbf{P}$ cnrichment in normally cutaneous trigeminal ganglion afferent projections during postnatal development. Direct target respecification of neurotransmitter phenotype is likely because stomach antrum transplants do not induce a change in the number of neurons comprising the projection. This target phenotype control of neurotransmitter expression in afferent projections can be dissociated from the postnatal remodeling of axon collateral projections in the same trigeminal neurons. The postnatal retraction of cerebral artery collaterals by single trigeminal neurons also possessing frontal nerve projections is not modified by the visceral or cutaneous targets innervated by the frontal nerve in the forehead. Rather, a difference in the ability of the visceral cerebral artery and the cutaneous forehead skin to support afferent innervation may induce trigeminal neurons to maintain forehead skin projections at the expense of cerebral artery collaterals.

\section{References}

Abercrombie M (1946) Estimation of nuclear population from microtome sections. Anat Rec 94:239-247.

Altman J, Bayer SA (1982) Development of the cranial nerve ganglia and related nuclei in the rat. Adv Anat Embry Cell Biol 74:1-90.

Baker H (1990) Calcitonin gene-related peptide in the developing mouse olfactory system. Dev Brain Res 54:295-298.

Barakat I, Droz B (1989) Inducing effect of skeletal muscle extracts on the appearance of calbindin-immunoreactive dorsal root ganglion cells in culture. Neuroscience $28: 39-47$.

Chan KY, Haske RH (1981) Action of a trophic factor(s) from rabbit corneal epithelial culture on dissociated trigeminal neurons. J Neurosci $1: 1155-1162$.

Chiaia NL, Hess PR, Rhoades RW (1987) Preventing regeneration of infraorbital axons does not alter the ganglionic or transganglionic consequences of neonatal transection of this trigeminal branch. Dev Brain Res 36:75-88.

Davies AM (1987) Molecular and cellular aspects of patterning sensory neurone connections in the vertebrate nervous system. Development 101:185-208. 
Davies AM, Lindsay RM (1984) Neural crest-derived spinal and cranial sensory neurons are equally sensitive to NGF but differ in their response to tissue extracts. Dev Brain Res 14:121-127.

Edvinsson L, Hara H, Uddman R (1989) Retrograde tracing of nerve fibres to the rat middle cerebral artery with true blue: colocalization with different peptides. J Cerebr Blood Flow Metab 9:212-218.

Erzurumla RS, Killackey HP (1983) Development of order in the rat trigeminal system. J Comp Neurol 213:365-380.

Gibbins IL, Furness JB, Costa M (1987) Pathway-specific patterns of coexistence of substance $P$, calcitonin gene-related peptide, cholecystokinin and dynorphin in neurons of the dorsal root ganglia of the guinea pig. Cell Tissue Res 248:417-437.

Green T, Dockray GJ (1987) Calcitonin gene-related peptide and substance $P$ in afferents to the upper gastrointestinal tract in the rat. Neurosci Lett 76:151-156.

Green T, Dockray GJ (1988) Characterization of the peptidergic afferent innervation of the stomach in the rat, mouse and guinea pig. Neuroscience 25:181-193.

Horgan K, O'Connor TP, van der Kooy D (1990) Prenatal specification and target induction underlie the enrichment of calcitonin gene-related peptide in the trigeminal ganglion neurons projecting to the cerebral vasculature. J Neurosci 10:2485-2492.

Katz DM, Erb MJ (1990) Developmental regulation of tyrosine hydroxylase expression in primary sensory neurons of the rat. Dev Biol 137:233-242.

Kessler JA, Black IB (1981) Nerve growth factor stimulates development of substance $P$ in the embryonic spinal cord. Brain Res 208: 135-145.

Kruger L, Silverman JD, Mantyh PW, Sternini C, Brecha NC (1989) Peripheral patterns of calcitonin gene-related peptide general somatic sensory innervation: cutaneous and deep terminations. J Comp Neurol 280:29l-302.

Lee Y, Shiosaka S, Takami K, Hillyard CJ, Girgis S, MacIntyre I, Emson PC, Tohyama M (1985) Coexistence of calcitonin gene-related peptide and substance P-like peptide in single cells of the trigeminal ganglion of the rat: immunohistochemical analysis. Brain Res 330: 194-196.

Lieberman AR (1974) Some factors affecting retrograde neuronal responses to axonal lesions. In: Essays on the nervous system (Bellairs R, Gray EG, eds), pp 71-105. Oxford: Clarendon.

Marusich MF, Weston JA (1988) Rolc of ncuron target interactions in the development of a subpopulation of avian sensory neurons. $J$ Neurosci Res 21:480-486.

Marusich MF, Pourmehr K, Weston JA (1986a) A monoclonal antibody (SN1) identifies a subpopulation of avian sensory neurons whose distribution is correlated with axial level. Dev Biol 118:494504.

Marusich MF, Pourmehr K, Weston JA (1986b) The development of an identified subpopulation of avian sensory neurons is regulated by interaction with the periphery. Dev Biol 118:505-510.

McMahon SB, Gibson S (1987) Peptide expression is altered when afferent nerves reinnervate inappropriate tissue. Neurosci Lett 73:915.

McMahon SB, Moore CEG (1988) Plasticity of primary afferent phosphatase expression following rerouting of afferents from muscle to skin in the adult rat. J Comp Neurol 274:1-8.

McMahon SB, Lewin GR, Anand P, Ghatei MA, Bloom SR (1989) Quantitative analysis of peptide levels and neurogenic extravasation following regeneration of afferents to appropriate and inappropriate targets. Neuroscience 33:67-73.

Molander C, Ygge J, Dalsgaard C-J (1987) Substance P-, somatostatinand calcitonin gene-related peptide-like immunoreactivity and fluoride resistant acid phosphatase-activity in relation to retrogradely labeled cutaneous, muscular and visceral primary sensory neurons in the rat. Neurosci Lett 74:37-42.

Nawa H, Patterson PH (1990) Separation and partial characterization of neuropeptide-inducing factors in heart cell conditioned medium. Neuron 4:269-277.

Nawa H, Sah DWY (1990) Different biological activities in conditioned media control the expression of a variety of neuropeptides in cultured sympathetic neurons. Neuron 4:279-287.

O'Brien C, Woolf CJ, ritzgerald M, Lindsay RM, Molander C (1989) Differences in the chemical expression of rat primary afferent neurons which innervate skin, muscle or joint. Neuroscience 32:493-502.

O'Connor TP, van der Kooy D (1986) Cell death organizes the post- natal development of the trigeminal innervation of the cerebral vasculature. Dev Brain Res 27:223-233.

O'Connor TP, van der Kooy D (1988) Enrichment of a vasoactive neuropeptide (calcitonin gene-related peptide) in the trigeminal sensory projection to the intracranial arteries. J Neurosci 8:2468-2476.

O'Connor TP, van der Kooy D (1989) Cooperation and competition during development: neonatal lesioning of the superior cervical ganglion induces cell death of trigeminal neurons innervating the cerebral blood vessels but prevents the loss of axon collaterals from the neurons that survive. J Neurosci 9:1490-1501.

Ositelu DO, Morris R, Vaillant C (1987) Innervation of facial skin but not masticatory muscles or the tongue by trigeminal primary afferents containing somatostatin in the rat. Ncurosei Lett 78:271276.

Philippe E, Droz B (1986) Effect of skeletal muscle on the expression of a calcium-binding protein (calbindin) in dorsal root ganglion of the chick embryo. Soc Neurosci Abstr 12:1125.

Philippe E, Droz B (1988) Calbindin D-28-immunoreactive neurons in chick dorsal root ganglion: ontogenesis and cytological characteristics of the immunoreactive sensory neurons. Neuroscience 26:215224.

Philippe E, Garosi M, Droz B (1988) Influence of peripheral and central targets on subpopulations of sensory neurons expressing calbindin immunoreactivity in the dorsal root ganglion of the chick embryo. Neuroscience 26:225-232.

Rao MS, Landis SC (1990) Characterization of a target-derived neuronal cholinergic differentiation factor. Neuron 5:899-910.

Rao MS, Landis SC, Patterson PH (1990) The cholinergic differentiation factor from heart cell conditioned medium is different from the cholinergic factors in sciatic nerve and spinal cord. Dev Biol 139: 65-74.

Ritter AM, Lewin GR, Kremer NE, Mendell LM (1991) Requirement for nerve growth factor in the development of myelinated nociceptors in vivo. Nature 350:500-502.

Saffran BN, Crutcher KA (1990) NGF-induced remodelling of mature uninjured axon collaterals. Brain Res 525:11-20.

Savy C, Margules S, Farkas-Bargeton E, Verley R (1981) A morphometric study of mouse trigeminal ganglion after unilateral destruction of vibrissae follicles at birth. Brain Res 217:265-277.

Shotzinger RJ, Landis SC (1988) Cholinergic phenotype developed by noradrenergic sympathetic neurons after innervation of a novel cholinergic target in vivo. Nature 335:637-639.

Shotzinger RJ, Landis SC (1990a) Postnatal development of autonomic and sensory innervation of thoracic hairy skin in the rat. Cell Tissue Res 260:575-587.

Shotzinger RJ, Landis SC (1990b) Acquisition of cholinergic and peptidergic properties by sympathetic innervation of rat sweat glands requires interaction with normal target. Neuron 5:91-100.

Smallheiser NR, Crain SM (1984) The possible role of "sibling neurite bias" in the coordination of neurite extension, branching, and survival. J Neurobiol 15:517-529.

Smith CL, Frank E (1987) Peripheral specification of sensory neurons transplanted to novel locations along the neuraxis. J Neurosci 7:15371549 .

Su HC, Wharton J, Polak JM, Mulderry PK, Ghatei MA, Gibson SJ, Terenghi G, Morrison JFB, Ballesta J, Bloom SR (1986) Calcitonin gene-related peptide immunoreactivity in afferent neurons supplying the urinary tract: combined retrograde tracing and immunohistochemistry. Neuroscience 18:727-747.

Terenghi G, Polak JM, Rodrigo J, Mulderry PK, Bloom SR (1986) Calcitonin gene-related peptide-immunoreactive nerves in the tongue, epiglottis and pharynx of the rat: occurrence, distribution and origin. Brain Res 365:1-14.

Tsai S-H, Tew JM, Shipley MT (1989) Cerebral artery innervation. II. development of calcitonin gene-related peptide and norepinephrine in the rat. J Comp Neurol 279:1-12.

Weston JA, Vogel KS, Marusich MF (1988) Identification and fate of neural crest cell subpopulations in early embryonic development. In: From message to mind (Easter SS Jr, Barald KF, Carlson BM, eds) pp 224-237. Sunderland, MA: Sinauer.

White FA, Bennett-Clarke CA, MacDonald GJ, Enfiejian HL, Chiaia NL, Rhoades RW (1990) Neonatal infraorbital nerve transection in the rat: comparison of effects on substance $P$ immunoreactive primary afferents and those recognized by the lectin Bandierea simplicifoliaI. J Comp Neurol 300:249-262. 